\title{
Adaptive Real-Time Dual-Mode Filter Design for Seamless Pedestrian Navigation
}

\author{
Pekka Peltola, Chris Hill and Terry Moore \\ Nottingham Geospatial Institute \\ Nottingham University, England \\ Email: \{pekka.peltola, chris.hill or terry.moore\}@nottingham.ac.uk
}

\begin{abstract}
Seamless navigation requires that the mobile device is capable of offering a position solution both indoors and outdoors. Novel seamless navigation system design was implemented and tested to achieve this aim. The design consists of general navigation system framework blocks and of the necessary interface agreements between the blocks. This approach enables plug-and-play style design of modules.

The implementation used four preselected key technologies. Microstrain 3DM-GX4-45 foot-mounted inertial measurement unit sensor data was fused together with the u-blox GNSS receiver positions outdoors. Context sensitive inference engine enabled the fusion of position updates indoors from the Decawave TREK1000 Ultra WideBand ranging kit and from the 6 Kontakt.io/Raspberry Pi anchor-based Bluetooth low energy fingeprinting system.

Novel dual-mode filter design uses a particle filter and the pentagon buffer enhanced Kalman filter in the position solution derivation. Depending on the map and the walls in the environment and on the quality of position updates, the implemented control logic employs the most fit filter for the current context. Computational power is now focussed, when particle filter is needed. The novel pentagon buffer enhanced Kalman filter is 10 times faster, allowing power saving when situation is not too critical. Moreover, the buffer provides position updates by interacting with the map and helps to correct the position solution.

The navigation system is seamless according to the tests conducted around and within the Nottingham Geospatial building. No user input is needed for smooth transition from outdoors to indoors and vice versa. The system achieves an accuracy of 2.35 $\mathrm{m}$ outdoors and $1.4 \mathrm{~m}$ indoors (95\% of error). Inertial system availability was continuous, while GNSS was available outdoors and BLE and UWB indoors.
\end{abstract}

\section{INTRODUCTION}

Great deal of interest is currently focussed on urban navigation. While car navigation is becoming mundane, pedestrian and smart environment based solutions are just on the verge of reaching the markets. Map and application design require efficient service from the underlying positioning sensor infrastructure and from the methods that are used in location acquisition. Processing power can be diverted to creating more user friendly interfaces when the navigation motor runs efficiently under the hood of a mobile device.

This paper introduces a novel approach for seamless navigation. Previous literature has mostly concentrated on enhancing a single filter type. The novel dual-mode filter presented here adapts to the current location and infrastructure by using two different types of filters. It is able to offer the most fit solution for the correct context by using the best sides of the particle and Kalman filters when needed. The efficiency of the method is proven by a campaign of test walks around the Nottingham Geospatial Building in the Jubilee Campus of the University of Nottingham.

Section II reviews the literature. Section III presents the novel system design. Section IV evaluates the implemented system which section $\mathrm{V}$ concludes.

\section{BACKGROUND}

We set the goals defined by Mautz in his Habilitation Thesis [1] for our adaptive system.

- Submeter level horizontal positioning accuracy

- Floor identification

- $>99 \%$ availability

- Minimal installation costs

Using optical tracking technology, the accuracy requirement is easily achieved. Santoso et al. discuss the visual inertial navigation technologies and methods in [2] with a special focus on microaerial vehicles. Issues common to visual and inertial navigation are examined like for example the brightness of the image. Time of flight cameras are mentioned, in which an additional depth information is available. An example of such is the camera on the Google Tango device. The camera approach is though far from being user-friendly and is computationally expensive, when considering navigation on devices for pedestrians. Requirement of continuous camera recording on a mobile device is not the most attractive alternative, which was the main reason why it was not included in the final design implementation.

Alarifi et al. examined the Ultra-wideband technology in detail in [3]. They list five main characteristics for a navigation system; accuracy/precision, coverage/resolution, latency, impact on infrastructure and random error resistance. Alarifi as well as Bras [4] agree the good sides using UWB being, the robustness to interference and jammers and high positioning accuracy due to large bandwidth. Antenna design for UWB systems is raised as an issue. Designing an antenna for an UWB system is not as easy as for narrowband systems.

Not many article research seamless multi-sensor navigation. Groves [5] comes perhaps closest. He lists four key challenges in multi-sensor navigation. These are complexity, context detection, ambiguous solutions and processing and storage capability. The key is to find the balance between performance 
and complexity. Moreover, best quality sensor data need to be sieved and bad quality discarded.

Yara et al. [6] developed a map interface with a purpose that it could be used as a plug-and-play map system for many kinds of navigation systems. Likewise $\mathrm{Xi}$ et al. [7] designed a sensor capable of adapting to different uses. NavCube was designed by Morrison et al. [8]. This was a platform that was capable of using four different GNSS receivers and had 10 slots for additional assisting sensors.

Along with the computational efficiency, accuracy is a desired feature of a navigation device. Sharp et al. [9] brings forward the geometric dilution of precision for circular and oval anchor placements. Rarely anchors within rooms indoors or satellites outside are in symmetric positions, but the study offers an insight into how to place anchors optimally.

User may be running, cycling or taking an elevator which all require readjustments of the navigation filter settings. These indicate different behavioral contexts. Location context depends on the estimated user position. In addition, virtual contexts like calendar events can be used in the inference of the best possible filter settings. System can also use learning methods for solution derivation if memory aspects are implemented.

Chen and Vadde [10] infer the context individually in the sensor submodules. The position information is then fed to the fusion motor with quality information. A centralized approach was used in our implementation, which means that no context is inferred in the sensor submodules.

Sensor output can be described by the level of abstraction [11]. Vertical abstraction describes the information content of the sensor output, which can be for example highly abstract sensor outputs like the calendar event in contrast to raw inertial data. Horizontal abstraction describes the capabilities of the similar types of sensors. One sensor measuring the same physical phenomena can output complementary information while another is capable of measuring the other end of the same phenomena scale.

As a part of the Marie Curie, Multi-Pos project, previous studies presented in [12], [13], [14] analyse the positioning technologies. On this basis, three absolute positioning technologies were selected. Global Navigation Satellite System (GNSS) receiver is responsible for outdoor positioning. UltraWideBand (UWB) and Bluetooth low energy (BLE) systems offer an absolute position indoors. Inertial measurement unit was mounted on the user's left boot for dead-reckoning the motion as an assisting relative positioning technology.

\section{AdAPTIVE DESIGN AND IMPLEMENTATION}

Figure 1 represents the adaptive design.

Sensor subsystems format the raw data for the Control and Navigation Blocks. In our design the data flow is only out of the Sensor Subsystems. The general format is following: \{timetag, measurement, error estimate\}. For GNSS subsystem, timetag is the system time of the mobile device, measurement is the LLH output provided by the sensor and error estimate is the sensor output estimate for horizontal accuracy. UWB least squares and BLE fingerprinting approaches are explained

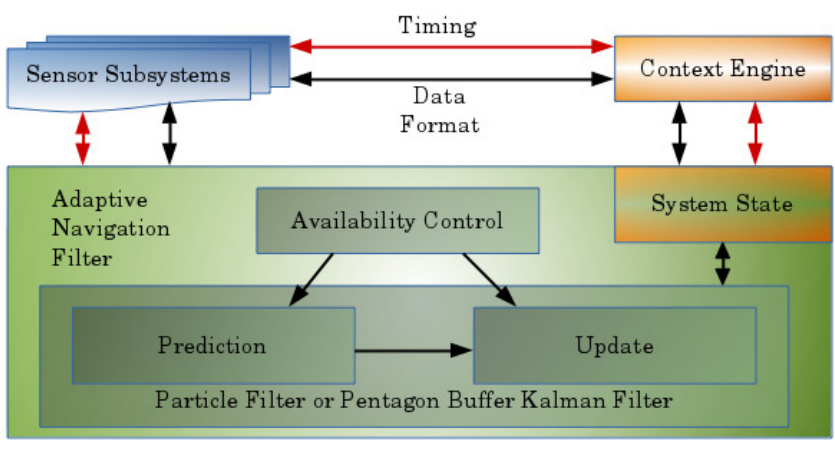

Fig. 1. Adaptive Dual-Mode Filter Design.

in [14]. Sensor subsystems blocks feed the navigation and control block according to the timing interface agreement. In our case, whenever a measurement is available it will be instantly delivered and processed.

The execution order starts from the sensor subsystems. When a measurement is ready the context engine is run first and the system state is set accordingly. Context engine also uses the estimated position information from the navigation filter together with the map sensor data. Finally, availability check infers the filter to be used by checking the system status and initiates either both the prediction and update cycles or just one that is needed. Availability block is in charge of data association, also by performing checks on the sensor subsystems data quality (e.g. deweigh if inside a wall).

\section{A. Context Engine}

Table I shows the implementation of the context engine. System status values are inferred as stated in the middle column.

TABLE I

CONTEXT ENGINE IMPLEMENTATION

\begin{tabular}{|c|c|c|}
\hline State flag & Inference & Values \\
\hline Position & From navigation filter & $(\mathrm{x}, \mathrm{y})$ \\
\hline ZUPT & ZUPT filter in Context Engine & TRUE/FALSE \\
\hline PF & $\begin{array}{c}\text { Pentagon Buffer fail/ Favourable } \\
\text { particle cloud properties }\end{array}$ & TRUE/FALSE \\
\hline Entrance & $\begin{array}{c}\text { Estimated position distance to entrance } \\
\text { <threshold value }\end{array}$ & TRUE/FALSE \\
\hline Stairs & Estimated position in the stairs & TRUE/FALSE \\
\hline Indoor & $\begin{array}{c}\text { Entrance flag AND approach direction is } \\
\text { between threshold values AND } \\
\text { GNSS/UWB/BLE availability check }\end{array}$ & TRUE/FALSE \\
\hline
\end{tabular}

Current estimated position is taken from the navigation filter. Zero update filter runs inside the Context Engine. This is a 7 tap (70ms delay) low pass filter for both the accelerometer and gyro total magnitudes. $100 \mathrm{~Hz}$ sampling frequency was used for the Microstrain 3DM-GX4-45 inertial measurements. High and low thresholds are used for accelerometer value and low threshold for gyro value. ZUPT flag is risen if accelerometer thresholds OR gyro threshold is exceeded.

Pentagon buffer fail logic and favourable particle cloud properties are explained later on. Entrance flag uses a single threshold value to indicate whether estimated position is close 
to the entrance. Stairs flag is turned on if estimated position is inside the stairs area defined in the map. Indoor flag status indicates whether indoor settings should be used for the particle and pentagon buffer filters. Entrance flag needs to be true and approach direction between threshold values for the current entrance. Final condition to switch the indoor flag is to check the measurement availability of the latest measurements. If only GNSS updates are available and horizontal accuracy has become better (threshold of $2.5 \mathrm{~m}$ ) these indicate a transfer to outdoors. Similarly if the GNSS horizontal accuracy values have been bad for longer time ( $3 \mathrm{~s}$ and horizontal accuracy over $2.5 \mathrm{~m}$ ) and availability of both UWB and BLE measurements show that they are present, the indoor flag is turned on.

\section{B. Particle Filter}

Basic bootstrap particle filter was used [15]. The dualmode implementation always started with particle filter turned on since it is considered to be the more robust filter. It can also handle the situtation better when initial values are unknown. Particle filter was initialised to the first position fix measurement. Direction initialisation distribution was uniform. After few steps the correct direction was achieved via particle elimination. Figure 2 lists the prediction and update steps for the particle filter.

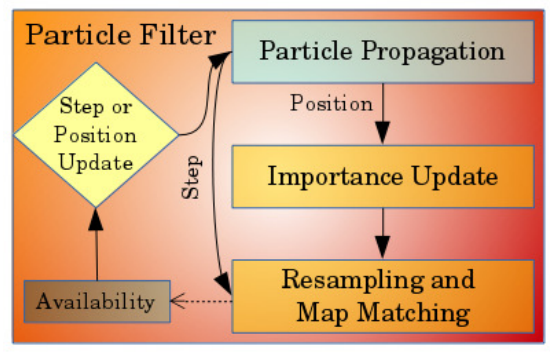

Fig. 2. Bootstrap particle filter.

Particle filter advances everytime either a step is detected or a position update from the absolute positioning systems (GNSS, UWB, BLE) is available. Particle consists of a position, direction and weight attributes. Propagation since the last particle filter update is estimated by propagating particles according to the IMU double integration mechanisation results. Small perturbation is added to each particle. Particle perturbation characteristics were derived experimentally. 20 short walks around the office were conducted. Two perturbation characteristics were derived from the data. Propagation angle perturbation was set to 1 degree per metre. Mechanisation length perturbation was set to $5 \mathrm{~cm}$ per metre. These values describe the estimated error of our inertial boot mounted system. For indoors these values were increased. This enhances particle filter survivability and compensates for errors that the perturbation addition phase is not able to handle.

Absolute positioning systems' position updates update the weight of each particle. For example, for each GNSS measurement the horizontal accuracy is given in Circular Error Probable (CEP, 50\% error bounds) which is used in the position update of each particle. Then the Bayesian inference step for the new value of each particle weight is derived according to the distance to the measurement.

Final step for the particle filter is the resampling step. We used resampling at every particle filter update to avoid impoverishment. Fixed ratio, $4 \%$ of particles were resampled each update cycle. Resampling was a transformation of dead particles into an inferred copy of survived particles. The more weight one particle had the more likely it was to be copied. Copying involved an addition of small perturbation in position and direction of the particle. Normalisation of the weights was the last step. If particle filter fails (that is every particle dies) a new particle cloud is created with wider seeding area at the previous update position and step is retaken.

\section{Kalman Filter with Pentagon Buffer}

Double integration mechanisation was implemented using a 15 error state closed loop Kalman filter explained in detail in Groves book [16]. This mechanisation filter ran continuously for the inertial mechanisation solution.

The Kalman filter state and covariance are copied into a parallel running solution that includes the position updates from GNSS, UWB and BLE. This means that the inertial Kalman solution runs as an unique solution. And in addition, when the particle filter is turned off, another Kalman solution takes care of the derivation of the final solution that includes position updates. The copy is created when particle filter is turned off the first time. This copy is then modified and used each time the particle filter is turned off.

Moreover, a pentagon buffer is created around the copied Kalman solution. This is shown in the left side of Figure 3.

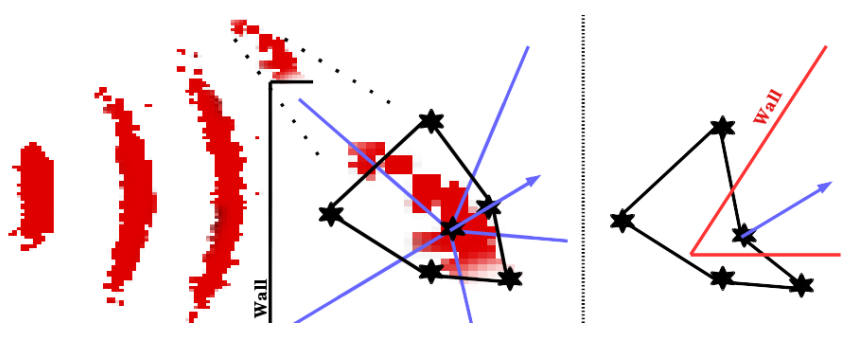

Fig. 3. On the left is shown the Particle filter switch into Pentagon buffer Kalman filter solution. On the right Pentagon buffer collision logic couldn't handle the collision and correct it with a position update and particle filter needs to be reinitialised.

\section{Particle filter to Pentagon Buffer switch}

The left side of Figure 3 depicts a switch of control from particle filter to the Kalman filter with pentagon buffer. Particle filter solution is the weighted average of the particles using their weight and position values. Particle cloud tends to change shape according to the walls and turns encountered. The possibility of a switch from particle filter to pentagon buffer is checked in the availability block. Main criteria for a switch is a threshold value for standard deviation of particles' distances from the main solution. If this is smaller than the threshold, Kalman filter is initialised. 
Buffer point distances, when initialised, are defined by the particle cloud properties. Particles are classified into five groups according to the sector they are in. The average of all the particles' directions of movements defines the sectoring angle. This is shown with a blue arrow in the Figure 3. The main solution is the centre point or the division point that divides the five sectors.

Distribution profile and 3-sigma limits are assigned for each sector. We used only Gaussian profile for simplicity. Using the standard deviation of the particles' distances from the main solution within each sector, a 3-sigma limit was derived. These were then the pentagon buffer point distances. This represents the maximum error inside which all of the solution probability should reside. More corners on the buffer would enhance the resolution but also increase the computation time. Buffer points are especially useful in the map matching phase.

\section{E. Pentagon Buffer propagation}

Buffer points represent the outer edges of the equivalent particle cloud. Thus it aids the Kalman filter to maintain a dynamic measure that is not included within the covariance. Each buffer point follows the main solution at its distance. The distances from the pentagon points to the main solution change according to walls, travelled distance and position update confidences. The distances are updated at every step and every position update. The experimentally defined position error growth, which was $5 \mathrm{~cm}$ per metre, is again used. In our study the same value was used for each direction. Direction dependent experimental error growth approximation could enhance the estimation of this buffer growth. Each buffer point distance is increased according to the position error growth and travelled distance.

Maximum limits for the buffer points' distances are defined by the position updates and are the 3-sigma limits for each position update measurement. The buffer point distances are reduced to this maximum limit if these start to grow too large.

After the propagation step, buffer points are matched with the map.

\section{F. Buffer logic and switch back to Particle filter}

Each buffer point is matched with the map. The walls act as reducing constraints to the buffer point distances. If a buffer point has propagated into a wall or has travelled through a wall its distance value is reduced until it no longer is inside a wall. If any of the distances goes below zero we have encountered a collision.

Collision logic then moves the solution towards the centre of the modified buffer until the solution is no longer inside the wall. If a collision, like what is depicted in the Figure 3 right side (none or only one buffer point distance is zero), happens, then the particle filter has to be reinitialised. The buffer logic is not able to apply a new unique position that is outside of the walls and complies with the direction of movement. The remaining area indicates two possible solutions (left and right side of the obstruction) where the user might have walked. Thus particle filter is reinitialised.
The switch is conducted using the covariance values of the Kalman filter and the latest position update measurement confidence values if available. New particles are seeded around the main Kalman solution. After this we conduct a map matching process that transforms particles that are inside the walls or outside of the remaining pentagon buffer into particle copies that are in the permitted area. This is the same process as was described in the particle filter section. We now have reinitialised the particle filter with particles inside the failed pentagon buffer limits.

\section{Evaluation}

UAV survey images were used for mapping and representing results in MATLAB. Four points were surveyed around the Jubilee Campus and the images were georeferenced in QGIS. Origin was the NGB12 survey point. The resulting images were then transferred to MATLAB.

C-program was written to record the Microstrain 3DMGX4-45 inertial and GNSS data, the Decawave TREK1000 kit UWB ranging data and BLE signal strengths. The resulting record files were used in MATLAB. Laptop that was used for analysing was running Debian on HP Stream x360. The designed system is straightforward to be used in real-time applications by replacing the file reader with real-time data transfer pipes.

Figure 4 shows the outdoor walk track. Altogether ten walks were conducted. 5 starting from the bicycle stands marked on the map. And another 5 walks along the reverse route, continuing from the main doors.

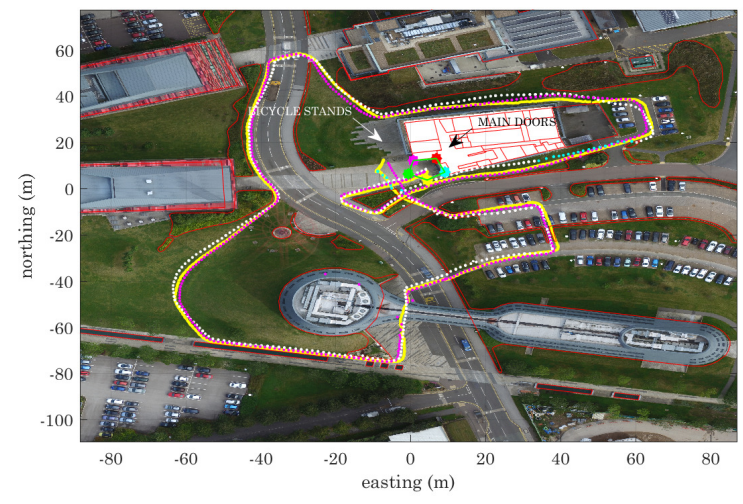

Fig. 4. Outdoor walking track. Magenta is the reference track recorded using the Leica RTK kit. Cyan track shows the particle filter solution. A record of the pentagon buffer is shown before entering indoors. White track shows the pentagon buffer Kalman filter track. Yellow track is the GNSS measurement trail.

In between, an indoor walk was conducted. Altogether five walks were conducted indoors and resemble the track shown in Figure 5.

The magenta track outdoors is the reference track recorded using Leica RTK kit. Yellow track is the track from U-Blox GNSS receiver inside the Microstain sensor. The cyan track is the Particle filter track when filter was initialised. Note that the GNSS cold start position is off by almost ten metres. White 


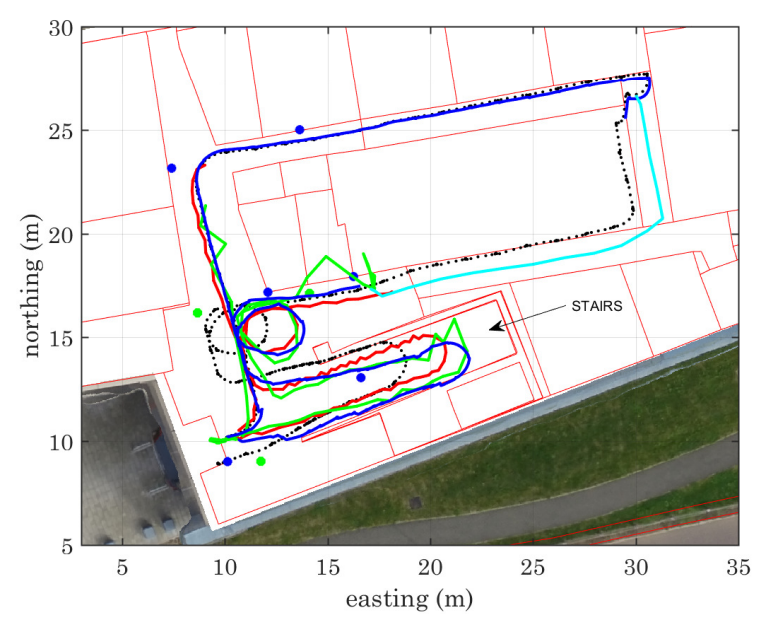

Fig. 5. Indoor walking tracks. Black track is the IMU only track. Green track is the UWB track and anchors. BLE anchors are in blue. Red track is the reference track recorded using Leica Total station. Blue track is the pentagon buffer track and cyan track show the particle filter track of the dual-mode filter.

track is the Pentagon Buffer track after control transfer from particle filter, in similar fashion to Figure 3. The fences on the walkway kill most of the particles and standard deviation of the particle cloud becomes small.

Black track is the inertial track indoors. Note the walker wobble in the northeast corner after opening the door. Red track is the Leica Total station reference track. Blue balls indicate the BLE anchor locations. Green balls and track are the UWB setup results. Blue track is the Pentagon buffer solution. In the northeast corner pentagon buffer fails and is switched to particle filter. Cyan track indicates the particle filter in the area where only IMU was available. Again control is switched back to Pentagon Buffer when particle cloud is suppressed by UWB measurements while entering lobby.

\section{A. On Accuracy}

The recorded data was processed first by using only particle filter. Then the same tracks were processed using the novel dual-mode filter design.

Figure 6 shows the accuracies for the UWB, BLE and particle and dual-mode filters. Inertial mechanisation had a tendency to swerve slightly right in our measurements. This means that an unknown heading error was present which turned the inertial solution more towards the right. Both particle filter position update and pentagon buffer error bound control were able to compensate this. Additional Kalman attitude updates would be helpful if there was a method to identify this error source.

It is also necessary to note here that the outdoor Kalman filter weight for GNSS position updates were weaker in comparison to the particle filter GNSS position updates. While particle filter solution followed the GNSS trace closely the pentagon buffer Kalman filter solution did this but with less weight in comparison. The GNSS receiver track was very accurate even alone. This is why the particle filter error was smaller. Though one walk had multipath error within the GNSS track. This can be seen in Figure 7. This track was not included in the accuracy Figure 6. Horizontal accuracy stayed good until the receiver, after 50 metres walk notices the multipath and fixes the position back to the walkway on the left side of the picture, where the user was actually walking. Weighting of the GNSS should be lowered drastically after the multipath condition is detected.

UWB is the most accurate. Performance degrades when walking away from the lobby (Figure 5). Indoors, particle filter and dual-mode filter performed similar $(1.4 \mathrm{~m}, 95 \%$ of error). Outdoors the weighting difference between particle and dual-mode filters for the good quality GNSS updates made the particle filter perform better $(2.35 \mathrm{~m}, 95 \%$ of error). BLE performance was not good and was given lower weight.

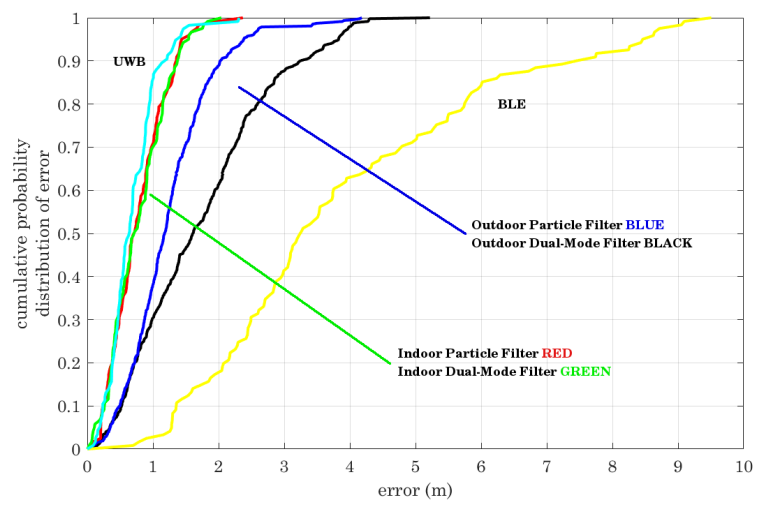

Fig. 6. Errors for technologies, UWB is cyan, BLE is yellow. Particle filter indoors is red, Dual-mode filter indoors is green, particle-filter outdoors is blue and dual-mode filter is black.

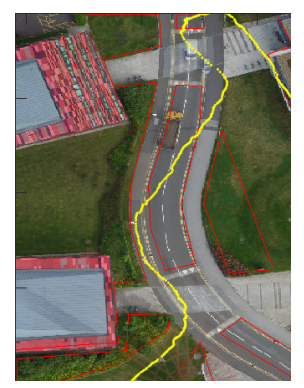

Fig. 7. Multipath affecting the GNSS receiver. Actual walk went along the left walkway.

Figure 8 describes how many particles were necessary to sustain reasonable accuracy for this particle filter setup. 50 and 100 particles accuracy graphs show degradation of accuracy. Thus 200 particles were used in our measurements.

\section{B. Dual-Mode Filter Advantages}

Figure 9 depicts the gain in speed when using dual-mode filter instead of particle filter. Computational load is ten times less for the pentagon buffer enhanced Kalman filter compared with the 200 particle filter. 


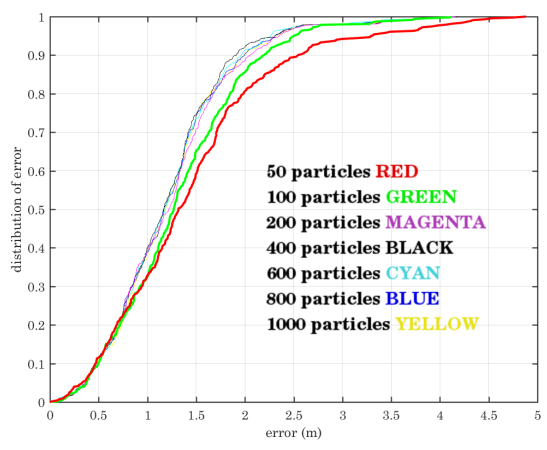

Fig. 8. Particle amount test for the particle filter shows that using over 100 particles will guarantee better accuracy.

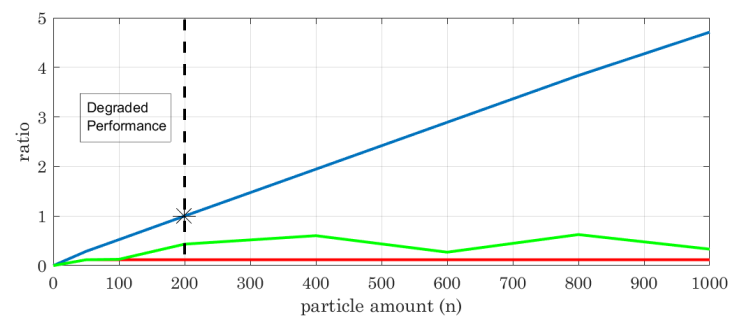

Fig. 9. 200 particles chosen as the reference (marked with star). Pentagon buffer alone runs 10 times faster (red line with 0.10 multiplier). Green is an example test run for the dual-mode filter with different amount of particles. If particle filter is on most the time, the green level is closer to the top blue line (only particle filter running).

\section{CONCLUSIONS}

Seamless navigation system was developed. The implementation works indoors, outdoors and is able to cope with the change of environment by using a simple context engine. Dual-mode operation uses particle filter when the pentagon buffer collision logic fails. Computation power is saved using pentagon buffer Kalman filter when conditions allow.

Submeter level accuracy was not yet achieved. Individual sensor technologies and fusion still need improvements. Floor identification was briefly examined by using IMU in the stairs. Availability outdoors is based on the GNSS availability. Although constantly available, inertial tracking is only accurate for few tens of meters after which the error is over a meter. UWB and BLE were deployed for the lobby and corridor areas. Wall constraints in corridors help the filter function with IMU only.

GNSS receiver that we used was a grade level higher quality than what is used in mobile phones. Quick tests conducted with an android mobile phone indicate performance closer to Figure 7. Multi-path condition indicator is necessary for the context engine to adjust the sensor fusion process. Short tests indicate that by matching the GNSS and IMU track forms, the multi-path condition could be inferred. Moreover this matching process could be used in position correction if the track qualities were known for both the inertial and GNSS tracks. In addition, a different position update approach was tested for the particle filter. Using a more loose measurement update distribution after multi-path condition is detected seems purposeful. Instead of the standard Gaussian measurement distribution update a 'Tukey'-style distribution could be used to compensate for the multi-path effect. This is nearly equal to weighing the GNSS less. These are left for future study.

\section{ACKNOWLEDGMENT}

This work was financially supported by EU FP7 Marie Curie Initial Training Network MULTI-POS (Multi-technology Positioning Professionals) under grant nr. 316528.

\section{REFERENCES}

[1] R. Mautz, Indoor Positioning Technologies, ETH Zurich, Department of Civil, Environmental and Geomatic Engineering, Institute of Geodesy and Photogrammetry (2012).

[2] F. Santoso, M.A. Garratt and S.G. Anavatti, Visual-Inertial Navigation Systems for Aerial Robotics: Sensor Fusion and Technology, IEEE Transactions on Automation Science and Engineering, vol. 14, no. 1, pp. 260-275, Jan. 2017.

[3] A. Alarifi, A. Al-Salman, M. Alsaleh, A. Alnafessah, S. Al-Hadhrami, M.A. Al-Ammar and H.S. Al-Khalifa, Ultra Wideband Indoor Positioning Technologies: Analysis and Recent Advances. Sensors 2016, 16, 707.

[4] L. Bras, N.B. Carvalho, P. Pinho, L. Kulas and K. Nyka, A Review of Antennas for Indoor Positioning Systems, International Journal of Antennas and Propagation, vol. 2012, Article ID 953269, 14 pages, 2012.

[5] P.D. Groves, L. Wang, D. Walter, H. Martin, K. Voutsis and Z. Jiang, The four key challenges of advanced multisensor navigation and positioning, 2014 IEEE/ION Position, Location and Navigation Symposium - PLANS 2014, Monterey, CA, 2014, pp. 773-792.

[6] C. Yara, Y. Noriduki, S. Ioroi and H. Tanaka, Design and implementation of map system for indoor navigation - An example of an application of a platform which collects and provides indoor positions, 2015 IEEE International Symposium on Inertial Sensors and Systems (ISISS) Proceedings, Hapuna Beach, HI, 2015, pp. 1-4.

[7] J. Xi, C. Yang, A. Mason and P. Zhong, Adaptive Multi-Sensor Interface System-On-Chip, 2006 5th IEEE Conference on Sensors, Daegu, 2006, pp. 50-53.

[8] A. Morrison, V. Renaudin, J.B. Bancroft, G. Lachapelle, Design and Testing of a Multi-Sensor Pedestrian Location and Navigation Platform. Sensors 2012, 12, 3720-3738.

[9] I. Sharp, K. Yu and Y.J. Guo, GDOP Analysis for Positioning System Design, in IEEE Transactions on Vehicular Technology, vol. 58, no. 7, pp. 3371-3382, Sept. 2009.

[10] K. Chen, K.R. Vadde, Design and evaluation of an indoor positioning system framework, UC Berkeley EECS Technical Report. UCB/EECS2016-16.

[11] K. Rein and J. Biermann, Your high-level information is my low-level data - A new look at terminology for multi-level fusion, Proceedings of the 16th International Conference on Information Fusion, Istanbul, 2013, pp. 412-417.

[12] A. Basiri, E.S. Lohan, P. Figueiredo e Silva, P. Peltola, C. Hill and T. Moore, Overview of positioning technologies from fitness-to-purpose point of view, International Conference on Localization and GNSS 2014 (ICL-GNSS 2014), Helsinki, 2014, pp. 1-7.

[13] A. Basiri, P. Peltola, P. Figueiredo e Silva, E.S. Lohan, T. Moore and C. Hill, Indoor positioning technology assessment using analytic hierarchy process for pedestrian navigation services, 2015 International Conference on Location and GNSS (ICL-GNSS), Gothenburg, 2015, pp. 1-6.

[14] P. Peltola, C. Hill and T. Moore, Particle filter for context sensitive indoor pedestrian navigation, 2016 International Conference on Localization and GNSS (ICL-GNSS), Barcelona, 2016, pp. 1-6.

[15] A.J. Haug. Bayesian Estimation and Tracking - A Practical Guide, Wiley, 2012.

[16] P.D. Groves, Principles of GNSS, Inertial, and Multisensor Integrated Navigation Systems, Second Edition, Artech House, 2013. 